\title{
Recognition of Plasmodium falciparum gametocyte surface antigens by plasma antibodies in asymptomatic Ghanaian school children
}

\author{
Bismarck Dinko*, Teun Bousema, Colin J Sutherland \\ From Challenges in malaria research \\ Basel, Switzerland. 10-12 October 2012
}

\section{Background}

Malaria transmission-reducing interventions are key components of malaria control and elimination [1]. However, little is known about the immune responses directed at circulating Plasmodium falciparum gametocytes in humans, knowledge of which would be useful in the development of anti-gametocyte vaccines, which would have the capability to reduce malaria transmission from humans to mosquitoes. In a study in the Gambia, mature gametocyte-infected erythrocytes of $P$. falciparum were found to carry antigens (gametocyte surface antigens, GSA) that were recognised by malaria patient's plasma antibodies. These anti-GSA antibodies, taken at a single timepoint, were weakly associated with lower duration of gametocyte carriage in these treated patients $[2,3]$. We then sought to determine longitudinal patterns in GSA antibody prevalence and its relationship to possible immune suppression of gametocyte carriage in vivo.

\section{Materials and methods}

Flow cytometry of cultured gametocyte-infected erythrocytes from 3D7 and from two recently adapted gametocyte-producung lines was used to detect and measure plasma antibodies recognising the erythrocyte surface. Plasma was obtained from asymptomatic $P$. falciparumpositive children attending school in a rainforest region in Ghana. These children were treated with dihydro-artemisinin piperaquine, and followed up weekly for 1 month.

\section{Results and conclusions}

By microscopy, 8.9\% (15/168) of the children enrolled carried gametocytes and a further $20 \%$ of them developed gametocytes during subsequent follow-up. (NASBA is also now being carried out to identify sub-microscopic gametocyte carriers.) Preliminary results from 113 samples tested in flow cytometry show that more than $50 \%$ of those in the sub-group of children with gametocytes at enrolment carry antibodies to GSA, and we expect this proportion to increase as gametocytes are developed during the followup. Further longitudinal flow cytometry, and NASBA analyses will enable us to understand the dynamics between immune responses to gametocytes and gametocyte carriage following treatment of asymptomatic malaria.

Published: 15 October 2012

\author{
References \\ Greenwood BM: Trends Parasitol 2008, 24:449-454. \\ Saeed M, et al: PLoS One 2008, 3:e2280. \\ Sutherland CJ: Mol Biochem Parasitoly 2009, 166:93-98.
}

doi:10.1186/1475-2875-11-S1-P24

Cite this article as: Dinko et al.: Recognition of Plasmodium falciparum gametocyte surface antigens by plasma antibodies in asymptomatic Ghanaian school children. Malaria Journal 2012 11(Suppl 1):P24. 